\title{
Chain-based Routing Model for Improving the Network Lifetime in WSNs
}

\author{
Byeongmu Shin ${ }^{1)}$, Jinsul Kim²)

\section{무선 센서 네트워크에서 네트워크 수명 향상을 위한 체인 기반의 라우팅 모델} \\ 신병무1), 김진술2)
}

\begin{abstract}
The basic premise of the Internet of Things is that smart sensors collaborate directly to provide new applications. In wireless sensor networks which is another foundational technology of the Internet of Things, interconnected intelligent wireless sensors sense the surroundings, collect data and transmit it to a sink. Since many applications in wireless sensor networks depend on the battery and it is difficult to charge the battery, it is very important to establish an energy efficient path to transmit the collected data to the sink. Therefore, the research to improve the network lifetime is one of important challenges in wireless sensor networks. In this paper, we propose a chain-based routing model for improving energy efficiency and network lifetime by reducing the long-distance data transmission of some nodes in wireless sensor networks. We compare the network life our proposed routing model with PEGASIS and CSS through simulations. The proposed routing model is better in terms of performance than PEGASIS and CCS.
\end{abstract}

Keywords: Chain Formation, Data Gathering, Routing Algorithm, Wireless Sensor Networks

$$
\text { 요 약 }
$$

사물 인터넷의 기본적인 전제는 스마트 센서가 직접 협업하여 새로운 응용프로그램을 제공하는 것이 다. 사물 인터넷의 또 다른 기본 기술인 무선 센서 네트워크에서 상호 연결된 다수의 지능형 무선 센 서가 주변 센싱을 통하여 데이터를 수집하고 싱크로 전송한다. 많은 무선 네트워크 응용프로그램이 배터리에 의존하며 배터리를 충전하는 것이 어렵기 때문에 싱크로 수집된 데이터를 전송하기 위해

Received(June 16, 2020), Review Result(1st: August 8, 2020, 2nd: September 24, 2020), Accepted(October 28, 2020)

1) (Department Head) 61186 Dept. of Electrical Electronics and Computer Engineering Graduate School of Industry and Technology, Chonnam National Univ., 77, Yongbong-ro, Buk-gu, Gwangju, Korea email: shin1899@lgcns.com

2) (Professor, Corresponding Author) 61186 School of Electronics and Computer Engineering, Chonnam National Univ., 77, Yongbong-ro, Buk-gu, Gwangju, Korea

email: jsworld@jnu.ac.kr 
에너지 효율적인 경로를 설정하는 것은 매우 중요하며 네트워크 수명을 향상시키기 위한 연구는 무 선 센서 네트워크에서 가장 중요한 과제 중 하나이다. 본 논문에서는 무선 센서 네트워크에서 일부 센서 노드의 장거리 데이터 전송을 줄여 센서 노드의 에너지 효율을 높이고 네트워크 수명을 향상시 키기 위한 체인 기반의 라우팅 모델을 제안한다. 제안하는 라우팅 모델은 작은 세그먼트 단위로 분할 된 센싱 영역을 기반으로 체인을 형성하고 데이터를 수집한다. 모의실험을 통하여 제안하는 라우팅 모델과 체인 기반 알고리즘으로 가장 많이 알려진 PEGASIS 및 CSS의 네트워크 수명을 비교 분석하 였다. 그 결과에서 제안하는 라우팅 모델의 네트워크 수명이 PEGASIS 및 CCS보다 우수함을 보여주 었다.

핵심어: 체인 형성, 데이터 수집, 라우팅 알고리즘, 무선 센서 네트워크

\section{1. 서론}

사물 인터넷(IoT: Internet of Things)은 스마트 센서, 센서 네트워크, 플랫폼, 서비스 응용 프로그램 과 사물을 연결해주는 통신망으로 구현 가능하며 광범위한 분야에 걸쳐 기존 서비스를 고도화시키 고 새로운 서비스를 위해 계획되고 있다[1][2]. 사물 인터넷의 네트워킹은 다른 장치를 센싱, 계산 및 통신할 수 있는 수많은 작은 지능형 센서들이 무선망으로 네트워크를 구성하여 상호 연결한다. 실제로, 무선 센서 네트워크 응용프로그램은 온-보드 배터리에 의존하며 에너지 공급이 제한되기 때문에 데이터 송.수신을 위한 에너지 소비 최소화의 관련 연구는 가장 중요한 과제 중 하나이다. 무선 센서 네트워크에서 센서 노드의 위치를 기반으로 체인 형성을 통하여 송·수신 거리를 줄이는 체인 기반의 알고리즘은 에너지 효율적인 측면에서 매우 효과적이다.

체인 기반 알고리즘으로 가장 많이 알려진 PEGASIS[3]는 그리드 알고리즘을 이용하여 인접 센 서와 체인을 형성하여 데이터 전송을 위한 에너지 소비를 줄였다. 그러나 체인의 루프를 방지하기 위하여 점차 체인에 추가되는 센서 노드 사이의 인접 거리가 증가하고 이러한 이유로 체인에서 일 부 노드는 이웃 노드에 데이터를 전송하기 위해 훨씬 더 많은 에너지를 소비하게 된다. PEGASIS 를 기반으로 네트워크 수명을 향상시키기 위해 제안된 다양한 알고리즘 [4-12] 중에서 CCS[4]는 센 싱 영역을 서로 다른 클러스터를 나타내는 원형 트랙으로 구분하고 각 트랙에서 체인을 형성하여 데이터를 수집하고 싱크에 가장 가까운 트랙의 헤드 노드로 전달되며 최종적으로 싱크로 전송하기 때문에 데이터를 전송할 수 있는 거리를 줄여 에너지를 절약할 수 있다. 그러나 싱크가 센싱 영역 에서 멀리 위치한 경우 싱크에 가장 가까운 트랙의 노드는 싱크로 데이터를 전송하기 위해 많은 에너지를 소비하게 된다. 본 논문에서는 이러한 문제 해결을 통하여 센서 노드의 에너지 효율을 높이고 네트워크 수명을 향상시키기 위하여 체인 기반의 라우팅 모델을 제안하고 모의실험을 통하 여 PEGASIS 및 CCS와 네트워크 수명을 비교 분석한다.

본 논문은 총 5 장으로 구성되며 2장에서는 잘 알려진 체인 기반 알고리즘을 살펴보고 3장에서는 무선 센서 네트워크에서 센서 노드의 에너지 효율을 높이고 네트워크 수명 향상을 위한 체인 기반의 라우팅 모델을 설명하고 4장에서는 제시한 라우팅 모델의 에너지 효율성 검증을 위해 모의실험을 통 하여 기존 알고리즘인 PEGASIS 및 CCS와 네트워크 수명을 비교 분석한다. 5장에서는 본 연구를 통해 얻은 결론에 대하여 논의한다. 


\section{2. 관련 연구}

본 장에서는 체인 기반 라우팅 알고리즘으로 가장 많이 알려진 PEGASIS와 PEGASIS 기반의 CCS 알고리즘을 설명한다.

\subsection{PEGASIS}

PEGASIS(Power-Efficient Gathering in Sensor Information Systems)[3]의 주요 아이디어는 각 센서 노드가 가까운 이웃과 통신하고 싱크로 전송하는 리더 노드의 역할을 번갈아 수행하여 센서 노드 의 에너지 소비를 균일하게 하는 것이다. PEGASIS는 네트워크에 대한 모든 노드의 글로벌 위치 정보를 기반으로 싱크에서 가장 먼 노드에서부터 그리드 알고리즘을 사용하여 체인을 형성한다. 각 라운드에 데이터 수집을 위해 토큰 전달 방식을 사용하여 [그림 1]과 같이 체인의 끝 노드로부 터 리더 노드 방향으로 수집된 데이터를 싱크로 전송하기 위해 리더 노드의 많은 에너지 소비를 고려하여 모든 센서 노드가 번갈아 리더의 역할을 수행한다. 그러나 PEGASIS는 그리드 알고리즘 을 사용하여 체인을 형성하고 체인에 포함된 노드의 재방문을 허용하지 않기 때문에 일부 노드는 가까운 인접 노드와 통신을 할 수 없게 되고 결국, 에너지 소비의 불균형을 초래한다.

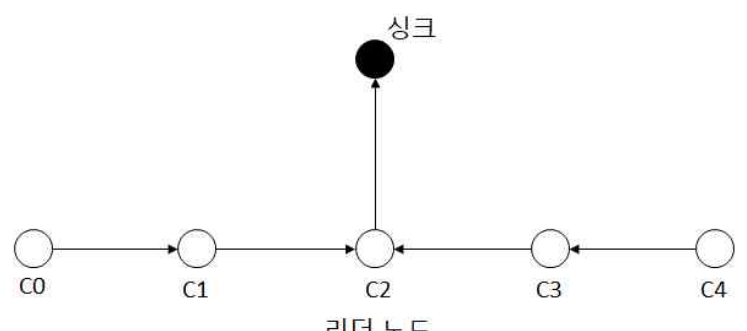

리더 노드

[그림 1] PEGASIS 데이터 전송

[Fig. 1] Data Transmission of PEGASIS

\subsection{CCS}

CCS(The Concentric Clustering Scheme for Efficient Energy Consumption in the PEGASIS)[4]는 센싱 영역을 서로 다른 클러스터를 나타내는 원형 트랙으로 구분하고 싱크에서 가장 가까운 트랙 부터 레벨을 증가하면서 할당한다. 체인은 각 트랙 영역에서 PEGASIS와 같은 방법으로 형성하고 형성된 체인에서 하나의 노드를 헤드로 선출(I $\bmod \mathrm{NL}, \mathrm{I}$ : 노드 $\mathrm{N}$ : 전체 노드 수 $\mathrm{L}$ : 레벨 번호)하 여 [그림 2]와 같이 자신의 위치 데이터를 상위 및 하위 레벨 헤드로 전송하여 싱크로부터 가장 가까운 레벨 1 헤드가 싱크로 데이터를 전송한다. CCS는 싱크가 센싱 영역에서 멀리 위치하고 있 
는 경우 하위 레벨에 분포하는 노드는 싱크로 데이터를 전송하기 위해 많은 에너지를 소비하는 단 점을 가지고 있다.

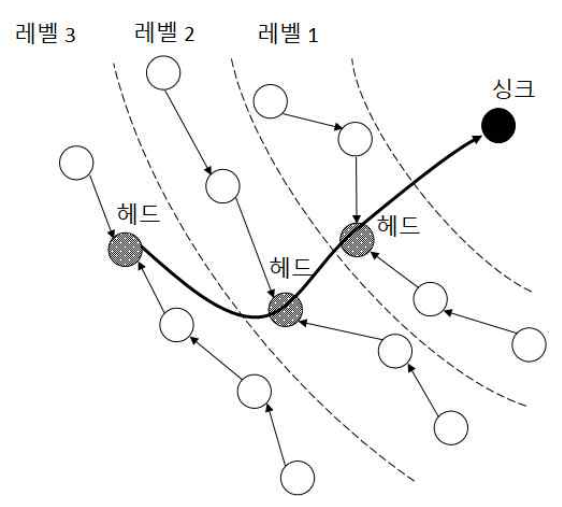

[그림 2] CCS 데이터 전송

[Fig. 2] Data Transmission of CCS

\section{3. 체인 기반의 라우팅 모델}

본 장에서는 제안하는 체인 기반의 라우팅 모델을 자세히 설명한다. 제안하는 라우팅 모델은 센싱 영역을 작은 세그먼트 단위로 분할하고 각 세그먼트를 기반으로 형성된 체인을 따라 데이터를 수 집하며 체인 형성 단계와 데이터 수집 단계로 구분한다.

\section{1 체인 형성 단계}

무작위로 센싱 영역에 배치된 센서 노드는 자신의 위치 정보를 싱크로 보내고 싱크는 수신한 위치 정보를 기반으로 최적의 네트워크 수명을 얻기 위한 허용수직거리를 정의한다. 본 논문에서는 허 용수직거리를 기준으로 센싱 영역을 분할하여 체인을 형성하기 때문에 허용수직거리 결정은 네트 워크 수명에 중요한 역할을 한다. 따라서 4장 성능분석에서는 제안하는 체인 기반의 라우팅 모델 에서 최적화된 네트워크 수명을 도출하기 위하여 허용수직거리를 다르게 정의하여 네트워크 수명 을 비교한다. 허용수직거리가 정의되면 센서 노드 중에서 [그림 3]과 같이 시작 노드와 종단 노드 를 결정하고 두 노드를 직선으로 연결하는 초기 기준선을 정의한다.
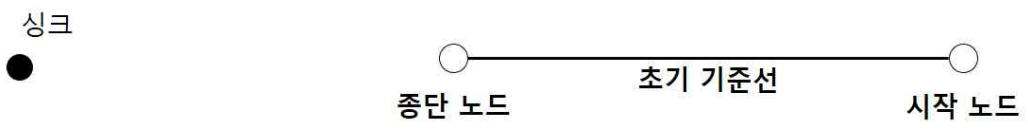

[그림 3] 시작 노드와 종단 노드 연결

[Fig. 3] Connection between Start and End Nodes 
시작 노드는 싱크에서 가장 먼 거리에 위치하는 노드이고 종단 노드는 시작 노드로부터 가장 먼 거리에 위치하는 노드이다. 초기 기준선으로부터 시작 노드와 종단 노드를 제외한 모든 노드의 수직 거리를 계산하여 가장 먼 수직거리의 노드를 수직 노드로 정의한다. 이때 초기 기준선과 수직거리를 기준으로 센싱 영역은 두 개의 영역으로 분할되며 [그림 4]와 같이 수직거리는 허용수직거리보다 크 지 않아야 한다. [그림 5]와 같이 시작 노드와 수직 노드 그리고, 수직 노드와 종단 노드를 각각 연 결하여 새로운 임시 기준선을 정의하고, 임시 기준선으로부터 허용수직거리보다 작은 수직거리에 위 치하는 수직 노드를 찾는 과정을 반복한다. 허용수직거리 이내에 센서 노드가 존재하지 않으면 시작 노드로부터 분할된 영역 내에서 탐욕 알고리즘을 사용하여 체인 형성을 시작한다. 한 분할 영역 내의 모든 노드가 체인에 연결되면 가장 가까운 거리의 수직 노드가 체인의 다음 노드가 되며 모든 노드 가 체인에 연결될 때까지 다음 노드의 선택을 계속한다.

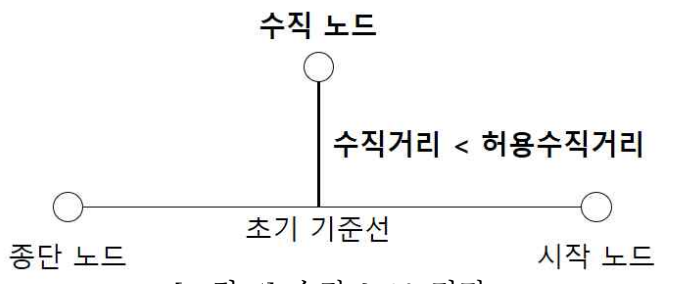

[그림 4] 수직 노드 결정

[Fig. 4] Decision of a Vertical Node

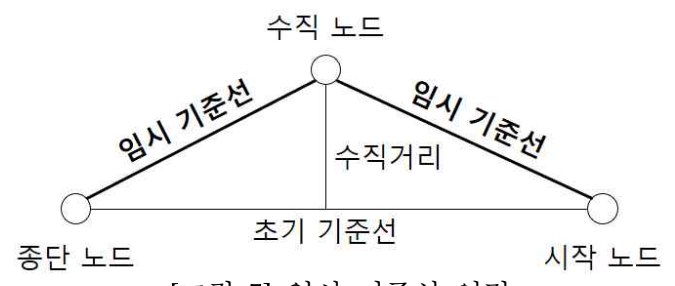

[그림 5] 임시 기준선 연결

[Fig. 5] Connection of Tentative Baseline

\section{2 데이터 수집 단계}

각 라운드에 수집된 데이터를 싱크로 전송하기 위하여 $\mathrm{r}$ 번째 라운드에 센서 노드 중 하나를 리더 노드로 선출한다(i $\bmod \mathrm{N}, \mathrm{i}$ : 노드 번호, $\mathrm{N}$ : 센서 노드 수). 이 방식을 통해 센싱 영역의 모든 노드는 체인 리더의 역할을 교대로 수행하며 [그림 6]과 같이 토큰 전달 방식을 사용하여 시작 노 드와 종단 노드로부터 체인의 리더 노드 방향으로 데이터를 수집한다.

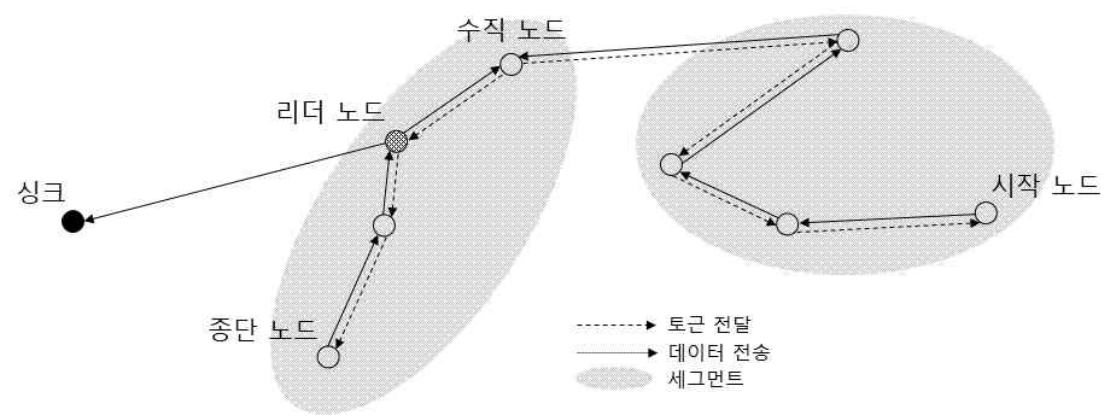

[그림 6] 토큰 전달과 데이터 전송

[Fig. 6] Token Passing and Data Transmission 
시작 노드와 종단 노드를 제외한 센서 노드는 인접 노드에서 수신한 데이터를 자신의 데이터와 융 합하여 체인을 따라 인접한 다음 노드로 전송하고 체인의 리더 노드는 일반적으로 인접한 두 노드로 부터 수신한 데이터를 융합하여 싱크로 전송한다.

\section{4. 성능 분석}

본 장에서는 모의실험을 통하여 제안하는 체인 기반의 라우팅 모델의 최적화된 수명을 도출하고 체인 기반 알고리즘으로 가장 많이 알려진 PEGASIS 및 PEGASIS 기반의 CCS와 네트워크 수명 을 비교 분석한다.

\section{1 모의실험 조건 및 가정}

본 논문에서 제안하는 체인 기반의 라우팅 모델의 네트워크 수명을 검증하기 위하여 Windows 환 경에서 $\mathrm{OMNeT}++[13][14]$ 를 사용한 모의실험의 모든 시나리오에서 제한된 에너지와 동일한 능력 을 가지고 있는 노드가 센싱 영역에 무작위로 배치되고 고정되며 각 노드는 자신의 위치를 알고 있다. 또한, 노드는 싱크로 데이터를 전송할 수 있으며 전송 범위를 조정할 수 있다. PEGASIS 및 $\mathrm{CCS}$ 와 동일한 조건에서 모의실험을 수행하였으며 [표 1]은 이러한 모의실험 조건을 보여준다. 메 시지 송·수신을 위해 PEGASIS 및 CCS에 사용된 것과 동일한 무선 모델을 [표 2]에 정의한다.

[표 1] 실험 조건

[Table 1] Parameters

\begin{tabular}{|c|c|c|c|}
\hline Parameter & Value & Parameter & Value \\
\hline Sensing Area & $100 \times 100 \mathrm{~m}^{2}$ & $E_{a m p}$ & $100 \mathrm{pJ} / \mathrm{bit} / \mathrm{m}^{2}$ \\
Sensor Nodes & $100,150,200,250$ & $E_{a g g}$ & $5 \mathrm{~nJ} / \mathrm{bit} / \mathrm{message}$ \\
Sink Location & $(50,300)$ & $k$ & $2000 \mathrm{bit}$ \\
$E_{\text {elec }}$ & $50 \mathrm{~nJ} / \mathrm{bit}$ & $E_{\text {init }}$ & $1 \mathrm{~J}$ \\
\hline
\end{tabular}

[표 2] 무선 모델

[Table 2] Radio Model

\begin{tabular}{|c|l|}
\hline Radio model & \multicolumn{1}{|c|}{ Formulas } \\
\hline \multirow{3}{*}{ Transmitting } & $E_{T X}(k, d)=E_{T X}-\operatorname{elec}(k)+E_{T X}-\operatorname{amp}(k, d)$ \\
\cline { 2 - 2 } & $E_{T X}(k, d)=E_{\text {elec }} \times k+E_{a m p} \times K \times d^{2}$ \\
\hline \multirow{2}{*}{ Receiving } & $E_{R X}(k)=E_{R X}-\operatorname{elec}(k)$ \\
\cline { 2 - 2 } & $E_{R X}(k)=E_{\text {elec }} \times k$ \\
\hline
\end{tabular}


$E_{T X}(k, d)$ 와 $E_{R X}(k)$ 는 각각 $k$ 비트 메시지를 $d$ 만큼의 거리로 전송하기 위한 에너지 소비와 $k$ 비 트 메시지를 수신하기 위한 에너지 소비를 계산하기 위한 식이다. $E_{e l e c}$ 은 전자 회로에서 신호를 송.수신하기 위하여 소비되는 에너지의 양이며 $a m p$ 는 증폭기에서 소비되는 에너지이다. $E_{T X}(k, d)$ 의 경우, 거리 $d$ 는 채널 전송으로 인한 $d^{2}$ 만큼의 에너지 손실을 가정한다.

\section{2 성능 분석 결과}

\subsection{1 제안하는 라우팅 모델의 네트워크 수명 분석}

본 논문에서 제안하는 체인 기반의 라우팅 모델의 최적화된 네트워크 수명을 찾기 위하여 허용수 직거리를 $20,30,40$ 으로 정의하고 각 허용수직거리에서 센서 노드의 수를 100, 150, 200 및 250으 로 증가시켜 첫 번째 장애 노드가 발생할 때까지 네트워크 수명을 측정하였다. [표 3]은 본 논문에 서 제안하는 라우팅 모델의 네트워크 수명을 측정한 결과이다. 센서 노드의 수와 허용수직거리가 각각 250 과 30 의 경우 본 논문에서 제안하는 라우팅 모델의 최적화된 네트워크 수명을 얻을 수 있 었다. PEGASIS 및 CCS와 네트워크 수명을 비교 분석하기 위하여 제안하는 라우팅 모델의 허용수 직거리를 30으로 정의하였다.

[표 3] 제안하는 라우팅 모델의 네트워크 수명

[Table 3] Network Lifetime of a Proposed Model

\begin{tabular}{|c|c|c|c|c|}
\hline & \multicolumn{4}{|c|}{ 센서 노드의 수 } \\
\cline { 2 - 5 } & 100 & 150 & 200 & 250 \\
\hline 허용수직거리(20) & 691 & 883 & 1182 & 1226 \\
허용수직거리(30) & 734 & 1197 & 1386 & 2349 \\
허용수직거리(40) & 961 & 954 & 1415 & 1760 \\
\hline
\end{tabular}

\section{2 .2 네트워크 수명 비교}

[그림 7]은 센서 노드의 수를 100, 150, 200 및 250으로 달리하여 본 논문에서 제안하는 라우팅 모 델(허용수직거리 30)과 PEGASIS 및 CCS의 네트워크 수명을 첫 번째 장애 노드가 발생할 때까지 측정한 결과를 나타내고 있다. 본 논문에서 제안하는 라우팅 모델(허용수직거리 30)은 PEGASIS 및 CCS보다 네트워크 수명이 약 2 배에서 5 배 더 효율적인 결과를 보인다.

이 결과에서 제안하는 라우팅 모델(허용수직거리 30)이 센싱 영역을 작은 세그먼트 단위로 분할하 고 분할된 센싱 영역을 기반으로 데이터 전송을 위한 체인을 형성하기 때문에 센서 노드 사이의 데 이터 전송 거리를 줄이는 효과와 에너지 소비의 균형을 이룰 수 있다는 것을 확인하였다. 다시 말해, 센서 노드가 데이터 전송을 위해 많은 에너지를 소비하기 때문에 장거리 통신은 네트워크 수명과 밀 접한 관련이 있다는 것을 확인할 수 있었다. 


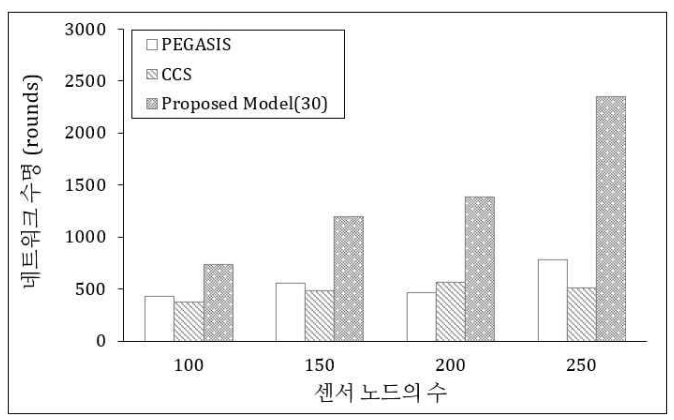

[그림 7] 네트워크 수명 비교

[Fig. 7] Comparison of the Network Lifetime

\section{5. 결론}

사물 인터넷 환경에서 무선망으로 네트워크를 구성하여 상호 연결된 다수의 지능형 센서는 주변 모니터링을 통하여 데이터를 수집한다. 실제로, 무선 센서 네트워크 응용프로그램이 배터리에 의존 하며 공급이 제한되기 때문에 데이터 송·수신을 위한 에너지 소비 최소화의 관련 연구는 가장 중 요한 과제 중 하나이다. 본 논문에서는 센싱 영역을 작은 세그먼트 단위로 분할하고 각 세그먼트 를 기반으로 형성된 체인을 따라 데이터를 수집하는 체인 기반의 라우팅 모델을 제안했다. 제안하 는 라우팅 모델의 목표는 무선 센서 네트워크에서 센서 노드의 에너지 소비 불균형을 개선하여 네 트워크 수명을 향상하는 것이다. 모의실험을 통하여 본 논문에서 제안하는 체인 기반의 라우팅 모 델의 최적화된 수명을 도출하고 체인 기반 알고리즘으로 가장 많이 알려진 PEGASIS 및 PEGASIS 기반의 CCS와 네트워크 수명을 비교 분석하였다. 그 결과 본 논문에서 제안하는 라우팅 모델의 네트워크 수명이 PEGASIS 및 CCS보다 우수함을 보여주었으며 이러한 결과를 바탕으로 향 후 무선 센서 네트워크에서 체인 기반의 알고리즘 연구에서 참고 자료로 이용될 것으로 기대된다.

\section{감사의 글}

본 연구 논문은 과학기술정보통신부 및 정보통신기획평가원의 대학ICT연구센터지원사업의 연구 결과로 수행되었으며(IITP-2020-2016-0-00314), 과학기술정보통신부 및 정보통신기획평가원의 출연금 등으로 수행하고 있는 한국전자통신연구원의 위탁연구과제(초연결 공통 네트워킹 서비스 연구인프 라 구축 및 2019-미래통신·전파-1)의 연구결과입니다.

\section{References}

[1] A. Al-Fuqaha, M. Guizani, M. Mohammadi, M. Aledhari, M. Ayyash, Internet of things: a survey on 
enabling technologies, protocols, and applications, IEEE Communications Surveys \& Tutorials, (2015), Vol.17, No.4, pp.2347-2376, DOI: 10.1109/COMST.2015.2444095

[2] P. Kamalinejad, C. Mahapatra, Z. Sheng, S. Mirabbasi, V. Leung, Y. L. Guan, Wireless energy harvesting for the internet of things, IEEE Communications Magazine, (2015), Vol.53, No.6, pp.102-108, DOI: 10.1109/MCOM.2015.7120024

[3] S. Lindsey, C. S. Raghavendra, PEGASIS: power efficient gathering in sensor information systems, Proceedings of IEEE Aerospace Conference, (2002), March 9-16; Big Sky, USA, DOI: 10.1109/AERO.2002.1035242

[4] S. M. Jung, Y. J. Han, T. M. Chung, The Concentric Clustering Scheme for Efficient Energy Consumption in the PEGASIS, The 9th International conference on Advanced Communication Technology, (2007), February 12-14; Gangwon, Korea, DOI: 10.1109/ICACT.2007.358351

[5] N. Tabassum, Q. Ehsanul, K. Mamun, Y. Urano, COSEN: A Chain Oriented Sensor Network for Efficient Data Collection, Third International Conference on information Technology: New Generations (ITNG'06), (2006), April 10-12; Las Vegas, USA, DOI: 10.1109/ITNG.2006.44

[6] J. E. Lee, K. Kim, Diamond-shaped routing method for reliable data transmission in wireless sensor networks, 2008 IEEE International Symposium on Parallel and Distributed Processing with Applications, (2008), December 10-12; Sydney, Australia, DOI: 10.1109/ISPA.2008.111

[7] I. Shukla, N. Meghanathan, Impact of leader selection strategies on the PEGASIS data gathering protocol for wireless sensor networks, Ubiquitous Computing and Communication Journal, (2009), Vol.4, No.5, pp.20-29.

[8] S. J. Lim, A. K. Bashir, S. Y. Rhee, M. S. Park, Energy based re-transmission algorithm of a leader node's neighbor node for reliable transmission in the PEGASIS, Proceedings of Ubiquitous Computing and Multimedia Applications(UCMA 2011), Vol.150, pp.120-128, (2011), April 13-15; Deajeon, Korea, DOI: https://doi.org/10.1007/978-3-642-20975-8_13

[9] Krishan Kumar, Deepti Gupta, Nirmal Kaur, Energy Energy Efficient Communication Protocol for Chain-based Clustered WSNs, International Journal of Grid and Distributed Computing, (2018), Vol.11, No.9, pp.81-92, DOI: $10.14257 /$ ijgdc.2018.11.9.07

[10] Aya Ayad Hussein, RajaaaldeenAbd Khalid, Improvements of PEGASIS Routing Protocol in WSN, International Advance Journal of Engineering Research, (2019), Vol.2, No.11, pp.1-14.

[11] Samayveer Singh, Pradeep Kumar Singh, A Clustering Based Optimized PEGASIS in Wireless Sensor Networks, International Conference on Futuristic Trends in Networks and Computing Technologies, (2019), November 22-23; Chandigarh, India.

[12] Fatima Es-Sabery, Abdellatif Hair, Evaluation and Comparative Study of the Both Algorithm LEACH and PEGASIS Based on Energy Consumption, Proceedings of the 3rd International Conference on Networking, Information Systems \& Security, (2020), March 31-April 2; Marrakech, Morocco, DOI: https://doi.org/10.1145/3386723.3387838

[13] https://www.omnetpp.org/, May 25 (2020)

[14] K. Wehrle, M. Gunes, J. Gross, Modeling and Tools for Network Simulation, Springer, (2010) 